\title{
COVID-19: implications for immunosuppression in kidney disease and transplantation
}

\author{
Andreas Kronbichler (10 ${ }^{\boxplus}$, Philipp Gauckler $\mathbb{D}^{1}$, Martin Windpess/2,3, Jae II Shin ${ }^{4,5,6}$, \\ Vivekanand Jha ${ }^{7,8,9}$, Brad H. Rovin ${ }^{10}$ and Rainer Oberbauer (iD ${ }^{11}$ \\ The coronavirus disease 2019 (COVID-19) pandemic poses important challenges to the care of \\ patients with immune-mediated kidney diseases and to kidney transplant recipients. Here, we \\ discuss the management of immunosuppression for these patients during the pandemic and \\ suggest potential approaches that could be considered in the absence of validated strategies.
}

Cellular immunity is key in determining the course and outcome of coronavirus disease 2019 (COVID-19) but is compromised in patients on immunosuppression, including transplant recipients and patients with glomerular diseases. A high proportion of patients with severe acute respiratory syndrome coronavirus-2 (SARS-CoV-2) infection (the cause of COVID-19) present with lymphopenia, and lymphocyte count has been negatively associated with disease severity ${ }^{1}$. Critically ill patients with COVID-19 exhibit high levels of cytokines, particularly IL-6, reinforcing the concept that hyperinflammation may contribute to morbidity and mortality in this disease and underscoring the delicate balance between protective and dysregulated host immune responses.

Immunosuppressive agents used in the management of glomerular diseases and kidney transplant recipients (Supplementary Table 1) have an inherent potential to cause lymphopenia and/or impair lymphocyte function. In the absence of specific therapy for SARS-CoV-2 infections, most nephrology societies have issued recommendations to reduce immunosuppression to levels that are considered safe, while acknowledging that balancing the risk of infections against the aim of disease control can be intricate even under 'normal' circumstances. However, such an approach is not feasible for patients with a de novo diagnosis of immune-mediated kidney disease, disease relapse or transplant rejection.

\section{Glomerular diseases}

No reports on treating glomerular diseases during the COVID-19 pandemic exist to date. Our suggestions are based on very little direct evidence but aim to balance the need to maintain viral host defence mechanisms against the need to suppress the immune system to treat the glomerular disease. The guiding principles of our suggestions are to avoid oversuppression of $\mathrm{T}$ cells and to consider the use of immunosuppressive drugs that may inhibit mechanisms of kidney injury and the cytokine storm that seems to be involved in the most severe cases of COVID-19.

Three clinical scenarios can be envisaged in patients with glomerulonephritis: first, either a new diagnosis or relapsing disease; second, the maintenance phase of immunosuppressive treatment; and third, long-term remission with ongoing tapering of immunosuppression. The decision to start immunosuppression in patients with newly diagnosed or relapsing glomerulonephritis and concomitant SARS-CoV-2 infection should ideally be based on kidney biopsy findings, kidney function, level of proteinuria, the expected tempo of the disease and comorbidities (Supplementary Table 2). If possible, treatment should be steered towards oral therapy to reduce in-person interactions with health-care personnel and avoid potential viral transmission and excessive use of personal protective equipment.

Despite ongoing controversies owing to a theoretical increased risk of SARS-CoV-2 infection and severe COVID-19 in patients using renin-angiotensin system (RAS) inhibitors, patients with proteinuria should be initiated on RAS inhibitors, as numerous recommendations support their continued use during the pandemic ${ }^{2}$. Initiation of immunosuppression should be considered in patients with diseases in which rapid deterioration of kidney function is expected and in those with severe nephrotic syndrome, which is accompanied by increased susceptibility to infections due to low IgG concentrations and depression of $\mathrm{T}$ cell function ${ }^{3}$.

In patients receiving maintenance immunosuppression, an individualized risk-benefit evaluation is necessary to assess the risk of immunosuppression versus the risk of glomerular disease relapse. If possible, patients who have not been exposed to SARS-CoV-2 should stay at home, and maintenance administration of drugs 
with long half-lives such as rituximab may be postponed until the current outbreak is under control. In the case of a clinic visit, we suggest that clinicians check markers of immunocompetence, such as white blood cells, differential blood count and immunoglobulin levels. Generally, we discourage prompt discontinuation of immunosuppression.

For patients in long-term remission, we encourage continued tapering of immunosuppression. If patients test positive for SARS-CoV-2 and have mild viral symptoms or are asymptomatic, we suggest reducing their glucocorticoid dose to $\leq 30 \mathrm{mg}$ daily and halting other immunosuppressive treatments that reduce lymphocyte numbers. If patients present with severe COVID-19, glucocorticoid use should likely be minimized to $20 \mathrm{mg}$ daily; however, such treatment decisions should be made on a case-by-case basis. As abrupt reduction or discontinuation of immunosuppression may result in a flare of glomerular disease, putting the kidney at risk or requiring intensification of immunosuppression, close follow-up of glomerulonephritis is required.

Among 701 patients with COVID-19 who were admitted to a hospital in Wuhan, China, $43.9 \%$ had proteinuria and $26.7 \%$ had haematuria ${ }^{4}$. This finding may point towards immune system activation during active SARS-CoV-2 infection, which may in turn increase the risk of relapse in patients with immune-mediated diseases. On the other hand, severe COVID-19 is often associated with severe lymphopenia, which could slow progression of immune-mediated glomerular disease if immunosuppression has to be temporarily discontinued.

No data are available on the safety of immunosuppressive treatments in patients with SARS-CoV-2 infections, and clinical practices are evolving. In patients with COVID-19 and active anti-glomerular basement membrane (anti-GBM) disease or severe anti-neutrophil cytoplasmic antibody (ANCA)-associated vasculitis (AAV), low doses of steroids ( $0.5 \mathrm{mg}$ per kg body weight) plus extracorporeal measures may be considered. Plasma exchange (PLEX) has a clear indication in anti-GBM disease and although the PEXIVAS trial failed to show long-term benefit of PLEX in AAV, it may be useful in the short term to mitigate AAV and allow reduced steroid doses ${ }^{5}$. PLEX should be performed against fresh frozen plasma, and convalescent plasma might be used

\footnotetext{
Author addresses

${ }^{1}$ Department of Internal Medicine IV (Nephrology and Hypertension), Medical University Innsbruck, Innsbruck, Austria.

${ }^{2}$ Department of Internal Medicine IV, Section of Nephrology, Klinikum Wels-Grieskirchen, Wels, Austria.

${ }^{3}$ Medical Faculty, Johannes Kepler University Linz, Linz, Austria.

${ }^{4}$ Department of Pediatrics, Yonsei University College of Medicine, Seoul, Republic of Korea. ${ }^{5}$ Division of Pediatric Nephrology, Severance Children's Hospital, Seoul, Republic of Korea. ${ }^{6}$ Institute of Kidney Disease Research, Yonsei University College of Medicine, Seoul, Republic of Korea.

${ }^{7}$ George Institute for Global Health, UNSW, New Delhi, India.

${ }^{8}$ George Institute for Global Health, University of Oxford, Oxford, UK.

${ }^{9}$ Manipal Academy of Higher Education, Manipal, India.

${ }^{10}$ Division of Nephrology, The Ohio State University Wexner Medical Center, Columbus, $\mathrm{OH}, \mathrm{USA}$.

${ }^{11}$ Department of Nephrology, Medical University of Vienna, Vienna, Austria.
}

at the end of the session if the patient has COVID-19. Immunoadsorption together with low-dose mycophenolate mofetil or calcineurin inhibitors (CNIs) and a reduced dosage of glucocorticoids might be considered in patients with active lupus nephritis ${ }^{6}$.

In AAV, immunomodulatory therapy with intravenous immunoglobulins is associated with a rapid reduction of disease activity and is an option for patients with COVID-19 (REF. ${ }^{7}$. Most studies in patients with AAV have found increased levels of IL- 6 and most other cytokines in blood and sites of active vasculitis, and treatment with tocilizumab, an IL-6 receptor inhibitor, is currently being investigated as potential therapy for this disease. Tocilizumab may also a promising agent for the management of cytokine release syndrome in COVID-19.

If rituximab is used for the management of glomerular disease, patients may not develop antibodies to SARS-CoV-2 and could be at risk of reinfection. Additionally, a poor vaccination response should be expected once vaccines against SARS-CoV-2 become available.

\section{Kidney transplant recipients}

Kidney allograft recipients require lifelong maintenance immunosuppression. Therapeutic drug monitoring is the standard of care to balance efficacy and safety, that is, prevention of an alloimmune response (rejection) at only a moderately increased risk of infections. As data on the treatment of SARS-CoV-2-positive kidney transplant recipients are lacking, it is fair to assume that reducing maintenance immunosuppression could be key to their management. What remains unclear, however, is the degree of immunosuppressant reduction that is required to enable SARS-CoV-2 containment. Post-transplant immunosuppression, in particular with CNIs, compromises the adaptive $\mathrm{T}$ cell response to a much greater extent than innate immunity and thus results in an increased risk of uncontrolled viral spreading and invasion. Non-immunosuppressive derivatives of ciclosporin decreased the expression of the $\mathrm{N}$ protein of human coronavirus 229E; this multifunctional protein is required for viral replication ${ }^{8}$. Based on these in vitro data, it might be speculated that ciclosporin could be used as the preferred CNI during the COVID-19 pandemic. No data are available on whether tacrolimus derivatives or metabolites exhibit similar in vitro activity.

Reports of COVID-19 in kidney transplant recipients are now emerging. In line with the experience in the general population, elderly transplant recipients are at increased risk of developing severe disease. Management of maintenance immunosuppression and initiation of antiviral, immunomodulatory and immunosuppressive therapies differ substantially between the published cases (Supplementary Table 3).

Based on the reported experience and good clinical practice extrapolated from other devastating courses of systemic viral infections, kidney transplant recipients with mild COVID-19 should continue to receive their CNIs and prescribed dose of glucocorticoids but their antiproliferative drugs should be stopped. In the minority of kidney allograft recipients with a severe course of 
confirmed COVID-19 requiring ICU admission and mechanical ventilation, CNIs and antiproliferative drugs should be immediately withdrawn and glucocorticoid doses should likely be increased. Whether anti-cytokine drugs such as anti-IL- 6 or complement inhibitors should be administered is currently unknown.

The WHO has launched a mega-trial (Solidarity, ISRCTN83971151) that will test four approaches for treatment of COVID-19: remdesivir; lopinavir-ritonavir; lopinavir-ritonavir with interferon- $\beta 1 \mathrm{a}$; and chloroquine or hydroxychloroquine (HCQ). Remdesivir was developed for the treatment of Ebola virus disease and works through blockade of the RNA-dependent RNA polymerase, a key viral enzyme, whereas lopinavirritonavir is an anti-HIV protease inhibitor drug combination. The first trial from Wuhan in which lopinavir-ritonavir was compared with standard care reported negative results ${ }^{9}$. In the Solidarity trial lopinavir-ritonavir will be tested alone or in combination with interferon- $\beta$ to control inflammation. Chloroquine and HCQ are antimalarial drugs that have been reported to exhibit antiviral activity against SARS-CoV-2 in vitro. Unusually, kidney transplant recipients are not excluded from the Solidarity trial and we hope that valid data will be available soon. Notably, some of the antiviral drugs exhibit significant drug-drug interactions, especially with CNIs, and thorough monitoring of the trough levels of ciclosporin or tacrolimus will be necessary. As for all clinical decisions, the characteristics of each individual patient need to be considered when making treatment decisions for COVID-19.

\section{Collateral damage}

Despite a lack of conclusive evidence of the efficacy of HCQ in combination with best supportive care for management of COVID-19, this drug is being used extensively for treatment and in some cases, prophylaxis. Some patients with lupus and rheumatoid arthritis are reportedly experiencing a HCQ shortage, increasing their risk of relapse. Owing to recommendations for 'physical distancing' to reduce the spread of SARS-CoV-2, outpatient appointments are being postponed in many countries and access to drug monitoring might be reduced owing to the strain on health-care facilities caused by the pandemic.
Likewise, transplantation programmes have been suspended in various countries because of scarce resources (especially ICU beds) and concerns regarding the unforeseeable consequences of common immunosuppressive induction regimens. In the USA, $71.8 \%$ of surveyed physicians reported full suspension of live donation kidney transplant programmes, and $80.2 \%$ of deceased donor kidney transplant programmes were operating with restrictions ${ }^{10}$. In Austria, the transplantation programme was reinitiated on 14 April 2020, but limited to kidney transplants (live and deceased donor) with low immunological risk that do not require anti-thymoglobulin or alemtuzumab induction regimens. If necessary, administration of anti-thymoglobulin for rejection or as part of the induction protocol should be minimized, given its profound effects on lymphocytes. In the current situation, our practice is that patients on the transplant waiting list need to sign a form stating that they are aware of the potential risks associated with transplantation during the COVID-19 pandemic.

In conclusion, medical evidence regarding optimal treatment approaches during this pandemic is currently limited. The suggestions discussed in this Comment remain to be validated in future studies.

1. Chen, G. et al. J. Clin. Invest. 130, 2620-2629 (2020).

2. South, A. M., Tomlinson, L., Edmonston, D., Hiremath, S. \& Sparks, M. A. Nat. Rev. Nephrol. https://doi.org/10.1038/ s41581-020-0279-4 (2020).

3. Hull, R. P. \& Goldsmith, D. J. BMJ 336, 1185-1189 (2008)

4. Cheng, Y. et al. Kidney Int. 97, 829-838 (2020).

5. Walsh, M. et al. N. Engl. J. Med. 382, 622-631 (2020).

6. Stummvoll, G. H. et al. Ann. Rheum. Dis. 64, 1015-1021 (2005)

7. Shimizu, T., Morita, T. \& Kumanogoh, A. Rheumatology 59, 959-967 (2019).

8. Ma-Lauer, Y. et al. Antiviral Res. 173, 104620 (2020).

9. Cao, B. et al. N. Engl. J. Med. 382, 1787-1799 (2020).

10. Boyarsky, B. J. et al. Early impact of COVID-19 on transplant center practices and policies in the United States. Am. J. Transplant. https://doi.org/10.1111/ajt. 15915 (2020).

\section{Competing interests}

The authors declare no competing interests.

\section{Peer review information}

Nature Reviews Nephrology thanks Annette Bruchfeld, Flavio Vincenti and the other, anonymous, reviewer(s) for their contribution to the peer review of this work.

\section{Supplementary information}

Supplementary information is available for this paper at https://doi.org/ 10.1038/s41581-020-0305-6. 